\section{RSP}

http://www.rsp.fsp.usp.br/
Revista de Saúde Pública

\title{
Integração entre os sistemas nacionais de informação em saúde: o caso do e-SUS Atenção Básica
}

\author{
Giliate Cardoso Coelho Neto' ${ }^{\text {iD }}$, Rosemarie Andreazza' iD, Arthur Chioro' \\ I Universidade Federal de São Paulo. Escola Paulista de Medicina. Programa de Pós-Graduação em Saúde \\ Coletiva. São Paulo, SP, Brasil
}

\section{RESUMO}

OBJETIVO: Medir o grau de integração do Prontuário Eletrônico do Cidadão (PEC) da Estratégia e-SUS Atenção Básica (e-SUS AB) com outros Sistemas Nacionais de Informação em Saúde (SNIS), o relacionando à estrutura político-organizacional interna do Ministério da Saúde (MS).

MÉTODOS: Trata-se de um estudo de caso de caráter qualitativo. A coleta de dados foi realizada através de análise documental e entrevistas semiestruturadas. Na primeira etapa buscou-se esclarecer quantos SNIS estiveram em uso na Atenção Básica do Sistema Único de Saúde entre 2013 e 2017. Em seguida, para medir a integração, foi aplicado como critério a manutenção das interfaces de captação de dados pelo Ministério da Saúde, mesmo após a implantação do PEC/e-SUS AB.

RESULTADOS: Foram identificados 31 SNIS na Atenção Básica. Observou-se que 12 deles

Correspondência:

Arthur Chioro

Universidade Federal de São Paulo

- Unifesp

Escola Paulista de Medicina Departamento de Medicina Preventiva

Rua Botucatu, n 740 - Vila Clementino

04023-062 São Paulo, SP, Brasil E-mail: arthur.chioro@unifesp.br

Recebido: 19 jul 2020

Aprovado: 26 dez 2020

Como citar: Coelho Neto FC, Andreazza R, Chioro A. Integração entre os sistemas nacionais de informação em saúde: o caso do e-SUS Atenção Básica. Rev Saude Publica. 2021;55:93. https://doi.org/10.11606/s15188787.2021055002931

Copyright: Este é um artigo de acesso aberto distribuído sob os termos da Licença de Atribuição Creative Commons, que permite uso irrestrito, distribuição e reprodução em qualquer meio, desde que o autor e a fonte originais sejam creditados. foram completamente integrados e em 15 não houve nenhuma unificação de interfaces com o PEC/e-SUS AB. Outros 4 tiverem integração parcial. Ao correlacionar esses dados com a estrutura político-organizacional do MS, verificou-se uma maior integração com os sistemas geridos pelo Departamento de Atenção Básica e uma persistência da fragmentação com os SNIS, especialmente aqueles sob gestão da Secretaria de Vigilância em Saúde. A disparidade entre a integração do PEC/e-SUS AB com os SNIS da Vigilância em Saúde é um sinal da persistência da divisão e da falsa dicotomia entre práticas e processos de Assistência à Saúde e Vigilância em Saúde no Ministério da Saúde - mesmo após 30 anos da fundação do SUS e unificação das estruturas estatais da assistência hospitalar previdenciária e da saúde pública federal no MS.

CONCLUSÃO: Apesar de ainda insuficiente, a integração de sistemas efetivada pela Estratégia e-SUS AB, que tem foco na redução de interfaces de usuário, pode ser considerada um fato novo na agenda da política de informação e informática do SUS.

DESCRITORES: Registros Eletrônicos de Saúde. Controle de Formulários e Registros. Políticas de eSaúde. Integração de Sistemas. Atenção Primária à Saúde. 


\section{INTRODUÇÃO}

Os profissionais, gestores e pesquisadores da área da saúde convivem com dezenas de sistemas de informação em saúde em seus ambientes de trabalho, com pouca ou nenhuma integração entre si. É um problema relatado desde a década de $1980^{1}$ e está relacionado à fragmentação das estruturas burocráticas do Estado ${ }^{2}$, à ausência de padronização semântica e tecnológica ${ }^{3-4}$ è baixa maturidade das políticas de governança de Tecnologia de Informação e Comunicação (TIC) nas organizações 5 .

Entre 2013 e 2018, foram identificados 54 Sistemas Nacionais de Informação em Saúde (SNIS) em funcionamento no Brasil, mantidos pelo Ministério da Saúde (MS), incluindo sistemas de cadastro, notificação de doenças e agravos, controle e logística de insumos e medicamentos, prontuários eletrônicos, gestão laboratorial, controle contábil da produção de procedimentos, dentre outros ${ }^{6}$. Ademais, incorporam-se a esses outros sistemas desenvolvidos ou adquiridos por estados e municípios.

Frequentemente, a pouca integração exige que os mesmos dados sejam preenchidos em diferentes interfaces, gerando retrabalho e aumento de custos, pois se torna necessário manter várias soluções tecnológicas com funções redundantes. Essa duplicidade de registros tem impacto na própria qualidade das bases de dados, dificultando as análises, os cruzamentos e impactando na confiabilidade da informação produzida ${ }^{4,7}$.

Desde a criação do Sistema Único de Saúde (SUS), algumas estratégias vêm sendo adotadas para melhorar a comunicação e integração entre os SNIS. Por iniciativa da Organização Pan-americana de Saúde, foi criada no final da década de 1990 a Rede Intergerencial de Informações para a Saúde (Ripsa), que tinha como objetivo produzir informações relevantes e integradas para a tomada de decisão na saúde ${ }^{8}$. A implantação do Cadastro Nacional de Usuário do SUS (Cadsus) nos anos 2000 se propunha a unificar as informações cadastrais básicas de todo cidadão brasileiro usuário do SUS. O primeiro modelo de gestão das informações do Cadsus previu o cadastramento, armazenamento e proteção dos dados como atribuições dos municípios, num modelo descentralizado que enfrentou graves problemas relacionados a cadastros duplicados, não sendo incomum a existência de múltiplos números do Cartão Nacional de Saúde (CNS) para o mesmo usuário. Isso acontecia quando o usuário comparecia a um serviço de saúde sem o seu cartão, exigindo que a recepção fizesse um novo cadastro para ele. Esse problema somente foi resolvido quando houve uma mudança estrutural na governança dos dados por meio da centralização das bases no MS. Ainda assim, em 2014, existiam apenas 14 SNIS conectados à base nacional do Cadsus 9 .

Em 2013, o MS lançou a Estratégia e-SUS Atenção Básica (e-SUS AB), que tinha como um de seus objetivos promover maior integração entre os SNIS em funcionamento nas Unidades Básicas de Saúde (UBS) ${ }^{10}$ por meio da unificação das interfaces de captação de dados nos softwares Coleta de Dados Simplificada (CDS) e no Prontuário Eletrônico do Cidadão (PEC), desenvolvidos em parceria com a Universidade Federal de Santa Catarina (UFSC) e disponibilizados gratuitamente às Secretarias Municipais de Saúde.

Segundo o MS, em 2016, o envio de dados para a base nacional do e-SUS AB era realizado por 97\% dos municípios brasileiros ${ }^{11}$ e o prontuário eletrônico já estaria instalado em 9.227 UBS, correspondendo a $21 \%$ das 42,6 mil existentes em $2017^{12}$. Esses números podem variar de acordo com a região e critérios utilizados. Por exemplo, Lima (2018) realizou estudo diagnóstico sobre a implantação da Estratégia e-SUS AB em Minas Gerais, concluindo que em apenas 49,1\% dos municípios a estratégia havia sido de fato implantada ${ }^{13}$. A par da controvérsia, parece inequívoco o avançado grau de implantação da política pública em questão.

Quando podemos afirmar que dois ou mais sistemas encontram-se integrados? No campo da Saúde Coletiva, esse entendimento geralmente esteve relacionado ao cruzamento de diferentes bases, aumentando a confiabilidade dos dados e produzindo novas informações em saúde ${ }^{14,15}$. Por sua vez, o debate sobre integração de sistemas no campo da informática em saúde tem 
acontecido em torno das soluções tecnológicas visando maior automação da comunicação entre sistemas (mediada por mensagens e arquivos padronizados, a interoperabilidade sintática) ou da compatibilização semântica entre os termos utilizados em cada um deles (a interoperabilidade semântica) ${ }^{16}$. Todavia, existe um outro aspecto da integração de sistemas que tem sido pouco abordado nas produções acadêmicas e nas políticas públicas de informação e informática em saúde, a chamada integração de interfaces de usuário ${ }^{17}$, relacionada ao design e usabilidade dos sistemas por trabalhadores e gestores nos serviços de saúde, responsáveis pela inserção dos dados primários nos sistemas.

Será a partir desse foco no utilizador-centrado que este artigo se propõe a descrever e medir a integração do PEC com outros SNIS da Atenção básica do SUS, o relacionando à estrutura político-organizacional do MS, testando a hipótese da relação da fragmentação de sistemas com a estrutura burocrática do Estado.

\section{MÉTODOS}

Realizou-se um estudo de caso que toma como objeto a relação entre a Estratégia e-SUS AB e os SNIS em uso na Atenção Básica, nos moldes propostos por Yin $(2015)^{18}$. Na primeira etapa, buscou-se esclarecer quantos SNIS estiveram em produção na Atenção Básica entre os anos 2013 a 2018 - necessário devido ao conflito existente entre as fontes de dados oficiais). Foram analisados os seguintes documentos do MS relacionados à informação e informática em saúde: Planos Diretores de Tecnologia da Informação (PDTI) dos anos de 2014 a 2015 ${ }^{19}, 2016^{20}$ e $20172018^{21}$; Política Nacional de Informação e Informática em Saúde (PNIIS) publicada em 201622; Relatório de Gestão do Datasus de 2011 a 2014²3; Termos de Referência dos processos referentes ao pregão eletrônico do contrato no 22/2014 referente à contratação de fábrica de software para prestação de serviços de tecnologia da informação para o desenvolvimento e a manutenção de sistemas de informação ${ }^{24} \mathrm{e}$, por fim, o Edital no $01 / 2017$ referente ao programa de informatização das Unidades Básicas de Saúde ${ }^{25}$.

Também foram analisadas as respostas do MS motivadas por dois pedidos de acesso à informação baseados na Lei no $12.527 / 2011$, onde foi perguntado sobre a lista de sistemas em produção no MS (Protocolo no 25820004082201795) e na Atenção Básica (Protocolo $n^{\circ}$ 25820005771201806). O conteúdo completo das respostas pode ser acessado de forma aberta na página do Sistema Eletrônico de Informações ao Cidadão (e-SIC)a .

Na segunda etapa, objetivou-se compreender a situação da integração de cada um dos SNIS em funcionamento na Atenção Básica com o Prontuário Eletrônico do Cidadão (PEC) do e-SUS $\mathrm{AB}$, esclarecendo se as ações de integração permitiram aos trabalhadores e gestores locais descontinuarem o uso do respectivo SNIS. Foram entrevistadas três informantes-chave, sendo dois membros da equipe de desenvolvimento e gestão da Estratégia e-SUS AB no período de 2013 a 2017 e um membro da equipe técnica de uma entidade nacional representativa das secretarias municipais de saúde. A análise contou também com contribuições da experiência de um dos pesquisadores na utilização cotidiana do PEC/e-SUS AB e de outros SNIS a partir de seu trabalho como médico em uma unidade básica de saúde do Rio de Janeiro. Considerou-se como SNIS integrado apenas aqueles onde houve consenso entre as fontes acima.

Foi criada a seguinte tipologia para classificar a situação da integração entre sistemas:

${ }^{a}$ Controladoria-Geral da União (BR). Sistema Eletrônico do Serviço de Informação (e-SIC) e Fala.BR - Módulo de Acesso à Informação: acesso à informação e ouvidoria em uma única plataforma. Brasília, DF; 2021 [citado 20 out 2021]. Disponivel em: http://esic.cgu.gov.br
- Interfaces de usuário integradas ou unificadas (integração completa): Quando dois ou mais sistemas se tornam invisíveis para o usuário final, ou seja, o usuário não consegue perceber que existe mais de um sistema em funcionamento, pois as interfaces estão totalmente integradas.

- Interfaces de usuário parcialmente integradas ou unificadas (integração incompleta): Quando foram identificadas iniciativas de integração entre as interfaces, mas ainda existe a necessidade, por motivos técnicos, políticos ou administrativos, dos profissionais e gestores continuarem utilizando os dois sistemas no cotidiano. 
Quadro. Sistemas Nacionais de Informação em Saúde (SNIS) que captaram dados na Atenção Básica entre 2013 a 2018 (excetuando-se os softwares da Estratégia e-SUS AB).

\begin{tabular}{|c|c|c|c|}
\hline Sigla & Nome do sistema & $\begin{array}{l}\text { Dado captado e/ou consumido ou principal } \\
\text { finalidade na } A B\end{array}$ & $\begin{array}{l}\text { Secretaria do } \\
\text { MS }\end{array}$ \\
\hline BFA & Sistema do Programa Bolsa Família na Saúde & $\begin{array}{c}\text { Registro da Antropometria de beneficiários do } \\
\text { Programa Bolsa família }\end{array}$ & SAS \\
\hline Cadsus & Sistema de Cadastramento dos Usuários do SUS & Cadastro dos usuários do SUS & SE \\
\hline CMD & Conjunto Mínimo de Dados & Registro de atividades e procedimentos ambulatoriais & SAS \\
\hline CNES & Cadastro Nacional dos Estabelecimentos de Saúde & Cadastro dos estabelecimentos de saúde & SAS \\
\hline Fique Sabendo & $\begin{array}{l}\text { Sistema de controle dos Testes Rápidos de HIV, } \\
\text { Sífilis e Hepatites Virais para ações estratégicas }\end{array}$ & Controle logístico dos testes rápidos & SVS \\
\hline GAL & Sistema de Gestão de Ambiente Laboratorial & $\begin{array}{l}\text { Visualização de Resultados de exames laboratoriais } \\
\text { para doenças de interesse de saúde pública }\end{array}$ & SVS \\
\hline Hiperdia & $\begin{array}{c}\text { Sistema de Cadastramento e Acompanhamento de } \\
\text { Hipertensos e Diabéticos }\end{array}$ & $\begin{array}{l}\text { Monitoramento de pacientes com Hipertensão } \\
\text { e Diabetes }\end{array}$ & SAS \\
\hline Hórus & Sistema Nacional de Assistência Farmacêutica & $\begin{array}{l}\text { Controle logístico de medicamentos do } \\
\text { componente básico da Política Nacional de } \\
\text { Assistência Farmacêutica }\end{array}$ & SVS \\
\hline PMAQ-AB & $\begin{array}{c}\text { Programa Nacional de Melhoria do Acesso e da } \\
\text { Qualidade da Atenção Básica }\end{array}$ & $\begin{array}{l}\text { Dados de ações programáticas e outras informações } \\
\text { da Atenção Básica para fins de avaliação das equipes }\end{array}$ & SAS \\
\hline Painel-PSE & Sistema de Adesão ao Programa Saúde na Escola & $\begin{array}{l}\text { Monitoramento de atividades realizadas pelas } \\
\text { equipes de Atenção Básica no âmbito do Programa } \\
\text { Saúde na Escola }\end{array}$ & SAS \\
\hline RAAS-AD & Registro das Ações de Saúde da Atenção Domiciliar & $\begin{array}{l}\text { Registro de procedimentos da atenção domiciliar no } \\
\text { âmbito do Programa Melhor em Casa }\end{array}$ & SAS \\
\hline RESP & Resposta a Eventos de Saúde Pública & Notificação de casos microcefalia & SVS \\
\hline SI-PNI & $\begin{array}{c}\text { Sistema de informação do Programa Nacional de } \\
\text { Imunização }\end{array}$ & $\begin{array}{l}\text { Monitoramento de Cobertura vacinal e controle } \\
\text { logístico de imunobiológicos }\end{array}$ & SVS \\
\hline SIA & Sistema de informações ambulatoriais & Registro de procedimentos ambulatoriais & SAS \\
\hline Siab & Sistema de Informação da Atenção Básica & Registro de atividades e procedimentos & SAS \\
\hline Siasi & Sistema de Informação da Saúde Indígena & $\begin{array}{l}\text { Demografia e acompanhamento clínico e } \\
\text { administrativos da população indígena }\end{array}$ & SESAI \\
\hline Siclom & $\begin{array}{c}\text { Sistema de controle e monitoramento clínico e } \\
\text { laboratorial dos pacientes em tratamento de hepatite }\end{array}$ & $\begin{array}{c}\text { Prescrição de medicamentos antirretrovirais para } \\
\text { tratamento de HIV/Aids }\end{array}$ & SVS \\
\hline SIM & Sistema de Informações sobre Mortalidade & Registro de óbitos naturais & SVS \\
\hline Sinan Net & Sistema de Informação de Agravos e Notificação & $\begin{array}{c}\text { Notificação de doenças e agravos de interesse da } \\
\text { saúde pública }\end{array}$ & SVS \\
\hline $\begin{array}{l}\text { Sinan Dengue/ } \\
\text { Chikungunya }\end{array}$ & $\begin{array}{c}\text { Sistema de Informação de Agravos de Notificação } \\
\text { para Dengue e Chikungunya }\end{array}$ & Notificação de casos de dengue e febre Chikungunya & SVS \\
\hline Sinan Influenza & $\begin{array}{l}\text { Sistema de Informação de Agravos e Notificação - } \\
\text { Influenza }\end{array}$ & $\begin{array}{l}\text { Notificação de casos de Síndrome Respiratória } \\
\text { Aguda Grave }\end{array}$ & SVS \\
\hline Sinasc & Sistema de Informações sobre Nascidos Vivos & Registro de nascidos vivos & SVS \\
\hline Siram & $\begin{array}{l}\text { Sistema de Registro de Atendimento às Crianças com } \\
\text { Microcefalia }\end{array}$ & $\begin{array}{l}\text { Registro de dados assistenciais sobre crianças com } \\
\text { diagnóstico de microcefalia }\end{array}$ & SAS \\
\hline Siscan & Sistema de Informação do Câncer & $\begin{array}{l}\text { Solicitação e resultados de pedidos de exames } \\
\text { relacionados ao diagnóstico de câncer }\end{array}$ & SAS \\
\hline Siscel & $\begin{array}{c}\text { Sistema de Controle de Exames Laboratoriais de CD4 } \\
\text { e Carga Viral }\end{array}$ & $\begin{array}{c}\text { Pedidos de exames de CD4 e Carga Viral de } \\
\text { pacientes com HIV }\end{array}$ & SVS \\
\hline Sisreg & Sistema Nacional de Regulação & $\begin{array}{l}\text { Solicitação e/ou marcação de procedimentos na } \\
\text { atenção especializada }\end{array}$ & SAS \\
\hline SIS Pré-natal & $\begin{array}{l}\text { Sistema de Informações de Monitoramento e } \\
\text { Avaliação do Pré-Natal, Parto, Puerpério e Criança }\end{array}$ & $\begin{array}{l}\text { Acompanhamento de gestante, puérperas e } \\
\text { recém-nascidos }\end{array}$ & SAS \\
\hline Sisvan & $\begin{array}{l}\text { Sistema de Vigilância e Acompanhamento } \\
\text { Nutricional }\end{array}$ & $\begin{array}{l}\text { Monitoramento do estado nutricional e } \\
\text { consumo alimentar }\end{array}$ & SAS \\
\hline Sivep DDA & $\begin{array}{c}\text { Sistema Informatizado de Vigilância Epidemiológica } \\
\text { de Doenças Diarreicas Agudas }\end{array}$ & Notificação de casos de Doença Diarreica Aguda & SVS \\
\hline
\end{tabular}


Quadro. Sistemas Nacionais de Informação em Saúde (SNIS) que captaram dados na Atenção Básica entre 2013 a 2018 (excetuando-se os softwares da Estratégia e-SUS AB). Continuação.

\begin{tabular}{|l|c|c|}
\hline Sivep Malária & $\begin{array}{c}\text { Sistema de Informação de Vigilância Epidemiológica } \\
\text { de notificação de casos de malária }\end{array}$ & Notificação de casos de Malária \\
\hline Telessaúde/Smart & $\begin{array}{c}\text { Sistema de Monitoramento e Avaliação dos } \\
\text { Resultados do Programa Telessaúde }\end{array}$ & $\begin{array}{c}\text { Controle, monitoramento e avaliação nacional de } \\
\text { programas de telessaúde }\end{array}$ \\
\hline
\end{tabular}

MS: Ministério da Saúde; BFA: Sistema do Programa Bolsa Família na Saúde; Cadsus: Sistema de Cadastramento dos Usuários do SUS; CMD: Conjunto Mínimo de Dados; CNES: Cadastro Nacional dos Estabelecimentos de Saúde; GAL: Sistema de Gestão de Ambiente Laboratorial; Hiperdia: Sistema de Cadastramento e Acompanhamento de Hipertensos e Diabéticos; Hórus: Sistema Nacional de Assistência Farmacêutica; PMAQ-AB: Programa Nacional de Melhoria do Acesso e da Qualidade da Atenção Básica; Painel-PSE: Sistema de Adesão ao Programa Saúde na Escola; RAAS-AD: Registro de Ações de Saúde da Atenção Domiciliar; RESP: Resposta a Eventos de Saúde Pública; SI-PNI: Sistema de informação do Programa Nacional de Imunização; SIA: Sistema de informações ambulatoriais; Siab: Sistema de Informações da Atenção Básica; Siasi: Sistema de Informação da Saúde Indígena; Siclom: Sistema de Controle e Monitoramento Clínico e Laboratorial dos Pacientes em Tratamento de Hepatite; SIM: Sistema de Informação sobre Mortalidade; Sinan Net: Sistemas de Informação de Agravos de Notificação; Sinan Dengue/Chikungunya: Sistema de Informação de Agravos de Notificação para Dengue e Chikungunya; Sinasc: Sistema de Informações sobre Nascidos Vivos; Siram: Sistema de Registro de Atendimento às Crianças com Microcefalia; Siscan: Sistema de Informação do Câncer; Siscel: Sistema de Controle de Exames Laboratoriais de CD4 e Carga Viral; Sisreg: Sistema Nacional de Regulação; SIS Pré-natal: Sistema de Informações de Monitoramento e Avaliação do Pré-Natal, Parto, Puerpério e Criança. Sisvan: Sistema de Vigilância e Acompanhamento Nutricional; Sivep DDA: Sistema Informatizado de Vigilância Epidemiológica de Doenças Diarreicas Agudas; Sivep Malária: Sistema de Informação de Vigilância Epidemiológica de notificação de casos de malária; Telessaúde/Smart: Sistema de Monitoramento e Avaliação dos Resultados do Programa Telessaúde. SAS: Secretaria de Atenção à Saúde; SE: Secretaria Executiva; SVS: Secretaria de Vigilância em Saúde; SGEP: Secretaria de Gestão Estratégica e Participativa; SESAI: Secretaria Especial de Saúde Indígena; SGTES: Secretaria de Gestão do Trabalho e Educação na Saúde; SCTIE: Secretaria de Ciência Tecnologia e Insumos Estratégicos.

Tabela 1. Situação de integração de interfaces de usuário do e-SUS AB com outros SNIS.

\begin{tabular}{|c|c|c|c|}
\hline $\begin{array}{l}\text { Situação da } \\
\text { integração }\end{array}$ & N SIS & $\%$ & Lista dos SIS \\
\hline Completa & 12 & $35,50 \%$ & $\begin{array}{c}\text { Cadsus, CMD, Hiperdia, PMAQ-AB, Painel-PSE, RAAS-AD, } \\
\text { SIA, Siab, Siram, SIS Pré-natal, Sisvan, BFA. }\end{array}$ \\
\hline Incompleta & 4 & $16,10 \%$ & CNES, Hórus, SI-PNI, Sisreg. \\
\hline Nenhuma & 15 & $48,40 \%$ & $\begin{array}{c}\text { Fique Sabendo, GAL, RESP, Siasi, Siclom, SIM, Sinan } \\
\text { Dengue/Chikungunya, Sinan Influenza, Sinan Net, Sinasc, } \\
\text { Siscan, Siscel, Sivep Malária, Sivep DDA, Telessaúde/Smart. }\end{array}$ \\
\hline
\end{tabular}

MS: Ministério da Saúde; BFA: Sistema do Programa Bolsa Família na Saúde; Cadsus: Sistema de Cadastramento dos Usuários do SUS; CMD: Conjunto Mínimo de Dados; CNES: Cadastro Nacional dos Estabelecimentos de Saúde; GAL: Sistema de Gestão de Ambiente Laboratorial; Hiperdia: Sistema de Cadastramento e Acompanhamento de Hipertensos e Diabéticos; Hórus: Sistema Nacional de Assistência Farmacêutica; PMAQ-AB: Programa Nacional de Melhoria do Acesso e da Qualidade da Atenção Básica; Painel-PSE: Sistema de Adesão ao Programa Saúde na Escola; RAAS-AD: Registro de Ações de Saúde da Atenção Domiciliar; RESP: Resposta a Eventos de Saúde Pública; SI-PNI: Sistema de informação do Programa Nacional de Imunização; SIA: Sistema de informações ambulatoriais; Siab: Sistema de Informações da Atenção Básica; Siasi: Sistema de Informação da Saúde Indígena; Siclom: Sistema de Controle e Monitoramento Clínico e Laboratorial dos Pacientes em Tratamento de Hepatite; SIM: Sistema de Informação sobre Mortalidade; Sinan Net: Sistemas de Informação de Agravos de Notificação; Sinan Dengue/ Chikungunya: Sistema de Informação de Agravos de Notificação para Dengue e Chikungunya; Sinasc: Sistema de Informações sobre Nascidos Vivos; Siram: Sistema de Registro de Atendimento às Crianças com Microcefalia; Siscan: Sistema de Informação do Câncer; Siscel: Sistema de Controle de Exames Laboratoriais de CD4 e Carga Viral; Sisreg: Sistema Nacional de Regulação; SIS Pré-natal: Sistema de Informações de Monitoramento e Avaliação do Pré-Natal, Parto, Puerpério e Criança. Sisvan: Sistema de Vigilância e Acompanhamento Nutricional; Sivep DDA: Sistema Informatizado de Vigilância Epidemiológica de Doenças Diarreicas Agudas; Sivep Malária: Sistema de Informação de Vigilância Epidemiológica de notificação de casos de malária; Telessaúde/Smart: Sistema de Monitoramento e Avaliação dos Resultados do Programa Telessaúde.

- Nenhuma integração ou unificação de interfaces de usuário: Quando não foi planejada nem realizada nenhuma ação para integração entre as interfaces dos sistemas.

Por fim, foi analisado o grau de integração dos SNIS com o organograma interno do MS. No período de 2013 a 2018, a estrutura formal do alto escalão do órgão se manteve estável, com a existência de sete Secretarias ${ }^{26}$ : Atenção à Saúde (SAS), Executiva (SE), Vigilância em Saúde (SVS), Gestão Estratégica e Participativa (SGEP), Especial de Saúde Indígena (SESAI), Gestão do Trabalho e Educação na Saúde (SGTES) e Ciência Tecnologia e Insumos Estratégicos (SCTIE). O Departamento de Atenção Básica (DAB), principal setor responsável pela formulação e gestão da Estratégia e-SUS AB, encontrava-se vinculado à SAS.

A pesquisa foi apresentada e aprovada no Comitê de Ética do Hospital São Paulo da Universidade Federal de São Paulo (Unifesp), por meio do parecer no 2.179.195. Os entrevistados assinaram Termo de Consentimento Livre e Esclarecido (TCLE) e as entrevistas foram realizadas e gravadas no software Skype ${ }^{\circledR}$. 
Tabela 2. Integração do e-SUS AB com outros SNIS por Secretaria do MS. Organograma de setembro de 2018.

\begin{tabular}{|c|c|c|c|c|}
\hline \multirow{2}{*}{ Secretaria do MS } & \multirow{2}{*}{$\begin{array}{l}\text { Total de SNIS em uso } \\
\text { na Atenção Básica }\end{array}$} & \multicolumn{3}{|c|}{ Integração de SNIS com o e-SUS AB } \\
\hline & & Completa & Incompleta & Nenhuma \\
\hline SAS & 14 & 11 & 2 & 1 \\
\hline SVS & 13 & 0 & 1 & 12 \\
\hline SE & 1 & 1 & 0 & 0 \\
\hline SCTIE & 1 & 0 & 1 & 0 \\
\hline SESAI & 1 & 0 & 0 & 1 \\
\hline SGTES & 1 & 0 & 0 & 1 \\
\hline SGEP & 0 & 0 & 0 & 0 \\
\hline TOTAL & 31 & 12 & 4 & 15 \\
\hline
\end{tabular}

SAS: Secretaria de Atenção à Saúde; SE: Secretaria Executiva; SVS: Secretaria de Vigilância em Saúde; SGEP: Secretaria de Gestão Estratégica e Participativa; SESAI: Secretaria Especial de Saúde Indígena; SGTES: Secretaria de Gestão do Trabalho e Educação na Saúde; SCTIE: Secretaria de Ciência Tecnologia e Insumos Estratégicos.

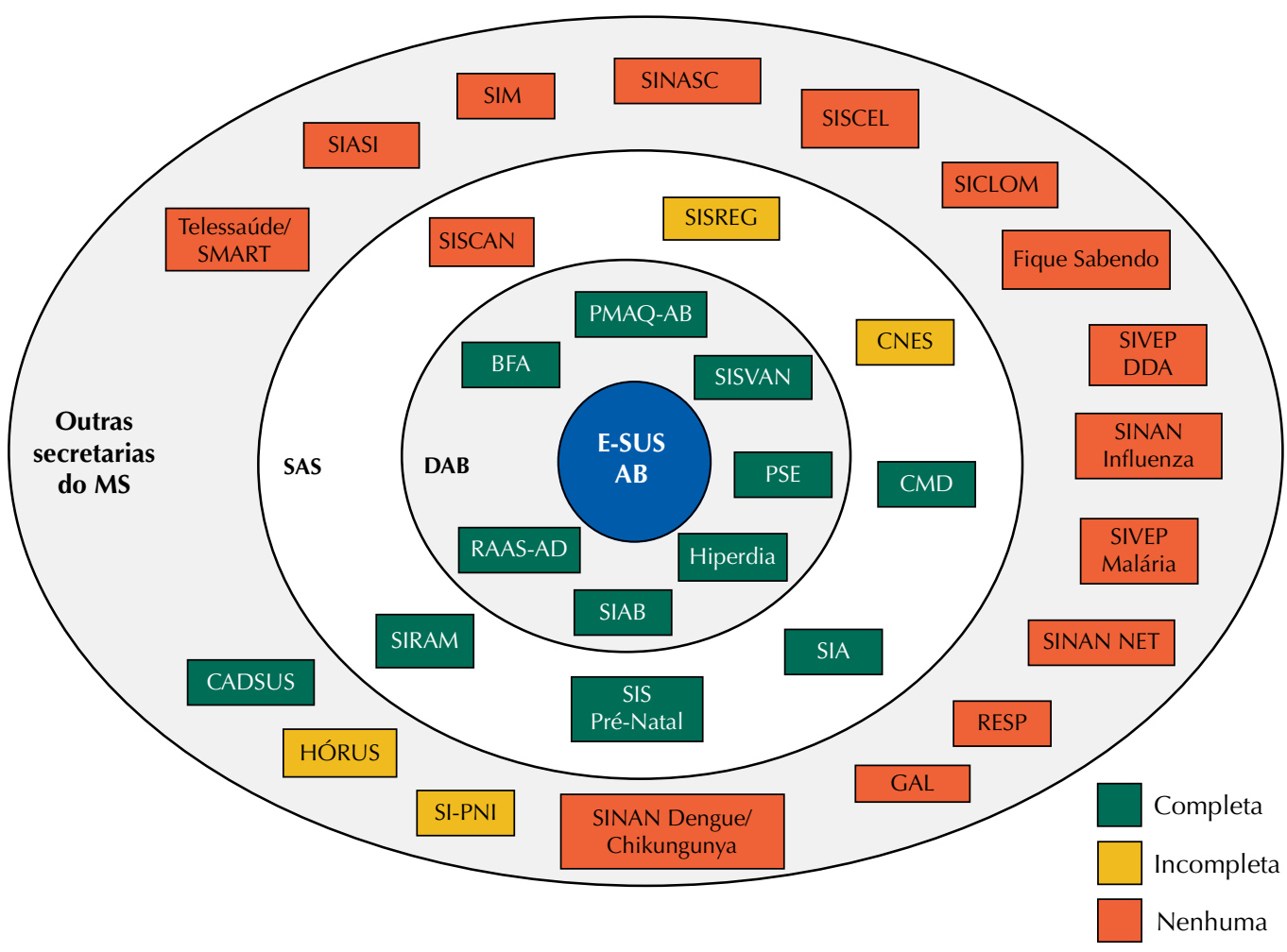

MS: Ministério da Saúde; SAS: Secretaria de Atenção à Saúde; DAB: Departamento de Atenção Básica; BFA: Sistema do Programa Bolsa Família na Saúde; Cadsus: Sistema de Cadastramento dos Usuários do SUS; CMD: Conjunto Mínimo de Dados; CNES: Cadastro Nacional dos Estabelecimentos de Saúde; GAL: Sistema de Gestão de Ambiente Laboratorial; Hiperdia: Sistema de Cadastramento e Acompanhamento de Hipertensos e Diabéticos; Hórus: Sistema Nacional de Assistência Farmacêutica; PMAQ-AB: Programa Nacional de Melhoria do Acesso e da Qualidade da Atenção Básica; Painel-PSE: Sistema de Adesão ao Programa Saúde na Escola; RAAS-AD: Registro de Ações de Saúde da Atenção Domiciliar; RESP: Resposta a Eventos de Saúde Pública; SI-PNI: Sistema de informação do Programa Nacional de Imunização; SIA: Sistema de informações ambulatoriais; Siab: Sistema de Informações da Atenção Básica; Siasi: Sistema de Informação da Saúde Indígena; Siclom: Sistema de Controle e Monitoramento Clínico e Laboratorial dos Pacientes em Tratamento de Hepatite; SIM: Sistema de Informação sobre Mortalidade; Sinan Net: Sistemas de Informação de Agravos de Notificação; Sinan Dengue/Chikungunya: Sistema de Informação de Agravos de Notificação para Dengue e Chikungunya; Sinasc: Sistema de Informações sobre Nascidos Vivos; Siram: Sistema de Registro de Atendimento às Crianças com Microcefalia; Siscan: Sistema de Informação do Câncer; Siscel: Sistema de Controle de Exames Laboratoriais de CD4 e Carga Viral; Sisreg: Sistema Nacional de Regulação; SIS Pré-natal: Sistema de Informações de Monitoramento e Avaliação do PréNatal, Parto, Puerpério e Criança. Sisvan: Sistema de Vigilância e Acompanhamento Nutricional; Sivep DDA: Sistema Informatizado de Vigilância Epidemiológica de Doenças Diarreicas Agudas; Sivep Malária: Sistema de Informação de Vigilância Epidemiológica de notificação de casos de malária; Telessaúde/Smart: Sistema de Monitoramento e Avaliação dos Resultados do Programa Telessaúde.

Figura. Integração de interfaces do e-SUS AB com SNIS utilizados na Atenção Básica conforme organograma interno do MS. 


\section{RESULTADOS}

Foram encontrados 31 SNIS em funcionamento na Atenção Básica entre 2013 e 2018, a maioria sob gestão técnica da SAS ou da SVS (Quadro).

Verificou-se que a Estratégia e-SUS AB realizou unificação completa de interfaces de usuário com 12 dos 31 SNIS de base nacional em uso na Atenção Básica e integrações incompletas com quatro SNIS. Outros 15 SNIS não tiveram nenhum grau de integração, conforme apresentado na Tabela 1.

A integração variou conforme o setor interno do MS responsável pela gestão técnica do sistema (Tabela 2). Apesar da SAS e SVS terem números próximos de SNIS em uso na Atenção Básica, verificou-se uma importante disparidade entre a integração dos seus respectivos sistemas com o e-SUS AB.

\section{DISCUSSÃO}

A fragmentação de SIS é um fenômeno relatado por diversos autores ao analisarem políticas de informação em saúde em diferentes regiões do mundo ${ }^{27-31}$, porém não foram identificados estudos que dimensionaram tal fragmentação a partir da contagem e descrição dos SNIS em funcionamento. O registro de 31 SNIS em uso na Atenção Básica no período de 2013 a 2018 evidencia que persiste no país a tendência de fragmentação, documentada no Brasil em diversos estudos técnicos ${ }^{32-34}$.

Nos países que apostaram em projetos de mudança desse cenário, o foco tem sido a melhor integração entre as bases de dados nacionai ${ }^{35-37}$ ou o estabelecimento de padrões semânticos e tecnológicos para registro e troca das informações entre diferentes sistemas ${ }^{38,39}$. Não foram encontrados estudos que descrevessem a integração de SNIS tendo como foco a integração de interfaces de usuário.

Quando analisamos o resultado da integração realizada pelo e-SUS AB e a estrutura político-organizacional do MS é possível notar que houve uma maior integração com os SNIS sob gestão direta do DAB (Figura) e uma baixa integração com sistemas das demais secretarias para além da própria SAS.

No caso da integração com os SNIS da SVS, que incluem as políticas de Vigilância Epidemiológica, de Atenção à População com HIV/Aids e Hepatites, de monitoramento da natalidade e mortalidade e de imunizações, houve avanço parcial apenas na integração do e-SUS AB com o SI-PNI.

O caso mais preocupante pareceu ser os SNIS do Departamento de HIV/Aids, que nem mesmo foram citados na lista de SNIS em funcionamento na Atenção Básica em licitação do MS para informatização de UBS ${ }^{25}$.

A disparidade entre a integração do e-SUS AB com os SNIS da SVS e da SAS pode ser considerada um sinal de que ainda persiste algum grau da histórica divisão e falsa dicotomia entre práticas e processos de Assistência à Saúde e Vigilância em Saúde ${ }^{40}$, mesmo após 30 anos da fundação do SUS e unificação das estruturas estatais da assistência hospitalar previdenciária e da saúde pública federal no MS.

A governabilidade da área gestora do e-SUS AB sobre outros SNIS se mostrou uma variável a ser considerada no processo de integração na medida em que houve uma maior taxa de sucesso de integração do e-SUS AB com os SNIS do próprio DAB quando comparada com os SNIS sob gestão de outros departamentos e secretarias do MS.

A frequência e intensidade do uso dos SNIS nos serviços de saúde demonstrou ser um fator de menor relevância, haja vista que os SNIS com uso intenso no nível local do SUS, como o Sisreg, Hórus e Sinan Net, tiveram integração incompleta ou não iniciada. 


\section{CONCLUSÃO}

Considerando a complexidade da integração entre sistemas legados nas organizações, é razoável que após cinco anos da implantação da Estratégia e-SUS AB, ele tenha se conectado apenas parcialmente com o total de SNIS em funcionamento na Atenção Básica. Apesar de ainda insuficiente, a integração de sistemas efetivada com cerca de 1/3 do SNIS em funcionamento da Atenção Básica, focada na redução de interfaces de usuário é um fato novo na agenda da política de informação e informática do SUS.

É notável a relativa singularidade da experiência brasileira de integração entre sistemas de informação em saúde. O Brasil apostou na criação de um prontuário eletrônico estatal como uma espécie de "hub" de outros SNIS criados pelo poder público, mais especificamente pelo Governo Federal. Apesar de consideramos que a postura "desenvolvimentista" assumida pelo Estado brasileiro contribui para a expansão da informatização da atenção básica no país, atentamos para a necessidade de maior integração do grande volume de software de mercado em utilização por estados e municípios nas bases nacionais dos SNIS, seguindo tendência de investimento em políticas de regulação pública associadas à maior interoperabilidade semântica e tecnológica entre os sistemas, conforme observado em outros países.

\section{REFERÊNCIAS}

1. Pinto LF, Freitas MPSA, Figueiredo AWS. Sistemas Nacionais de Informação e levantamentos populacionais: algumas contribuições do MS e do IBGE para a análise das capitais brasileiras nos últimos 30 anos. Cienc Saude Coletiva. 2018;23(6):1859-70. https://doi.org/10.1590/1413-81232018236.05072018

2. Moraes IHS, Gómez MNG. Informação e informática em saúde: caleidoscópio contemporâneo da saúde. Cienc Saude Coletiva. 2007;12(3):553-65. https://doi.org/10.1590/S1413-81232007000300002

3. Jardim SVB. The Electronic Health Record and its contribution to healthcare information systems interoperability. Procedia Technol. 2013;9:940-8. https://doi.org/10.1016/j.protcy.2013.12.105

4. Laguardia J, Domingues CMA, Carvalho C, Lauerman CR, Macário E, Glatt R. Information system for notifiable diseases (Sinan): challenges in developing a national health information system. Epidemiol Serv Saude. 2004;13(3):135-46. https://doi.org/10.5123/S1679-49742004000300002

5. Gottschalk P. Maturity levels for interoperability in digital government. Gov Inf Q. 2009;26(1):75-81. https://doi.org/10.1016/j.giq.2008.03.003

6. Coelho Neto GC. Integração entre Sistemas de informação em Saúde: o caso do e-SUS Atenção Básica [dissertação]. São Paulo: Escola Paulista de Medicina, Universidade Federal de São Paulo; 2019.

7. Araújo YB, Rezende LCM, Queiroga MMD, Santos SR. Sistemas de Informação em Saúde: inconsistências de informações no contexto da Atenção Primária. J Health Inform. 2016;8(5):164-70.

8. Risi Júnior JB. Informação em saúde no Brasil: a contribuição da Ripsa. Cienc Saude Coletiva. 2006;11(4):1049-53. https://doi.org/10.1590/S1413-81232006000400025

9. Ministério da Saúde (BR), Departamento de Informática do SUS. Interoperabilidade Sistema-SUS: apresentação. Brasília, DF; 2014 [citado 4 dez 2018]. Disponível em: https://portalarquivos2.saude.gov.br/images/pdf/2015/maio/15/2b-Apresentacao-WebServicesCONASSEMS.pdf

10. Rezende FAVS, Soares MF, AC. Os sistemas de informação em saúde no Sistema Único de Saúde. In: Leandro BBS, Rezende FAVS, Pinto JMC, organizadores. Informações e registros em saúde e seus usos no SUS. Rio de Janeiro: Editora Fiocruz; 2020. p.70-117.

11. Ministério da Saúde (BR); Conselho Nacional dos Secretários Municipais de Saúde. Acompanhamento e-SUS AB: nota informativa. Brasília, DF; 2016 [citado 12 maio 2017]. Disponível em: https://www.conasems.org.br/wp-content/uploads/2016/02/images_E_SUS.pdf 
12. Lora V. Funcionalidades do Prontuário Eletrônico do Cidadão. Rio Claro, SP-SP; 2018 [citado 4 dez 2018]. Apresentação realizada no $32^{\circ}$ Congresso de Secretários Municipais de Saúde do Estado de São Paulo. Disponível em: http://www.cosemssp.org.br/congresso/wpcontent/uploads/2018/05/VANESSA-LORA-FUNCIONALIDADES-DA-ESTRAT\%C3\%89GIA-eSUS-AB.pptx

13. Lima PKM. Implementação da Estratégia e-SUS Atenção Básica em municípios mineiros [dissertação]. São João Del-Rei, MG: Universidade Federal de São João Del-Rei; 2018.

14. Morais RM, Costa AL. Uma avaliação do Sistema de Informações sobre Mortalidade. Saude Debate. 2017;41 No Espec:101-17. https://doi.org/10.1590/0103-11042017S09

15. Oliveira C, Oliveira LCS, Guimarães MJB, Lyra T. Integração dos bancos do SIM e do SINAN: a contribuição da vigilância dos óbitos relacionados às doenças de notificação compulsória no município de Recife. In: Anais da $5^{\text {a }}$ EXPOEPI - Mostra Nacional de Experiências Bem-sucedidas em Epidemiologia, Prevenção e Controle de Doenças; 2- 6 dez 2005; Brasília, DF. Brasília, DF: Ministério da Saúde; c2006. p. 95.

16. Moreno RA. Interoperabilidade de Sistemas de Informação em Saúde [editorial]. J. Health Inform. 2016 [citado 4 dez 2018];8(3):I. Disponível em: http://www.jhi-sbis.saude.ws/ojs-jhi/ index.php/jhi-sbis/article/viewFile/502/268

17. Nebe K, Zimmermann D. Aspects of Integrating User Centered Design into Software Engineering Processes. In: Jacko JA, editor. Human-computer interaction. Interaction Design and Usability; Part I, HCl 2007. Berlin (DE): Springer-Verlag; 2007. p. 194-203. (Lecture Notes in Computer Science; $\mathrm{n}^{\circ}$ 4550). https://doi.org/10.1007/978-3-540-73105-4_22

18. Yin RK. Estudo de caso: planejamento e métodos. 2. ed. Porto Alegre: Bookman; 2013.

19. Ministério da Saúde (BR). Plano Diretor de Tecnologia da Informação - PDTI 2014-2015. Brasília, DF; 2013.

20. Ministério da Saúde (BR). Plano Diretor de Tecnologia da Informação - PDTI 2016. Brasília, DF; 2016.

21. Ministério da Saúde (BR). Plano Diretor de Tecnologia da Informação - PDTI 2017-2018. Brasília, DF; 2017.

22. Ministério da Saúde (BR), Secretaria Executiva, Departamento de Monitoramento e Avaliação do SUS. Política Nacional de Informação e Informática em Saúde. Brasília, DF; 2016.

23. Ministério da Saúde (BR), Departamento de Informática do SUS. Datasus: Relatório Executivo da Gestão 2011-2014. Brasília, DF; 2015.

24. Ministério da Saúde (BR). Termo de Referência da Licitação nº 0019/2013 (Processo administrativo $n^{\circ}$ 25000.090683/2012-51). Objeto: Pregão eletrônico - contratação de empresa especializada para prestação de serviços técnicos de desenvolvimento e manutenção de sistemas de formação; e contagem de pontos de função. Brasília, DF; 2017.

25. Ministério da Saúde (BR), Secretaria Executiva, Subsecretaria de Assuntos Administrativos, Coordenação Geral de Material e Patrimônio. Edital de credenciamento n ${ }^{\circ} 01 / 2017$ (Processo Administrativo $n^{\circ}$ 25000.072832/2017-13). Objeto: Contratação de empresas para implantação de prontuário eletrônico nas Unidades Básicas de Saúde (UBS), por meio da contratação de solução que contemple serviços de hardware, software, manutenção de equipamentos de TI, treinamento dos profissionais de saúde e suporte técnico para o uso do Prontuário Eletrônico, conforme especificações do presente Projeto Básico e de seus apêndices, para atendimento ao Ministério da Saúde. Brasília, DF; 2017.

26. Ministério da Saúde (BR), Secretaria Executiva, Departamento de Monitoramento e Avaliação do SUS. Planejamento Estratégico do MS 2011-2015: resultados e perspectivas. Brasília, DF; 2013 [citado 4 dez 2018]. Disponível em: https://bvsms.saude.gov.br/bvs/publicacoes/planejamento_ estrategico_ministerio_saude_resultados.pdf

27. Mudaly T, Moodley D, Pillay A, Seebregts C. Architectural frameworks for developing national health information systems in low and middle income countries. In: Proceedings of the 1st International Conference on Enterprise Systems, ES, 2013; 2013 Nov 7-8; Cape Town, South Africa. p.1-9. https://doi.org/10.1109/ES.2013.6690083

28. World Health Organization. Health Metrics Network: framework and standards for country health information systems. 2. ed. Geneva (CH): WHO; 2008 [citado 4 dez 2018]. Disponível em: http://www.who.int/healthmetrics/documents/hmn_framework200803.pdf

29. Kaushik A, Raman A. The new data-driven enterprise architecture for e-healthcare: lessons from the Indian public sector. Gov Inf Q. 2015;32(1):63-74. https://doi.org/10.1016/j.giq.2014.11.002 
30. Karuri J, Waiganjo P, Orwa D, Manya A. DHIS2: the tool to improve health data demand and use in Kenya. J Health Inform Dev Ctries. 2014 [citado 4 dez 2018];8(1):38-60. Disponível em: https://www.jhidc.org/index.php/jhidc/article/view/113

31. Mahundi M, Kaasbøll J, Twaakyondo H. Health Information Systems Integration in Tanzania: tapping the contextual advantages. In: Proceedings of the IST Africa Conference; 11-13 May 2011; Gaborone, Botswana. p.1-11.

32. Moraes IHS. Informação em saúde: da prática fragmentada ao exercício da cidadania. Rio de Janeiro: Hucitec;1994.

33. Ministério da Saúde; Organização Pan-Americana da Saúde; Fundação Oswaldo Cruz. A experiência brasileira em sistemas de informação em saúde. Vol. 1, Produção e disseminação de informações sobre saúde no Brasil. Brasília, DF; 2009. (Série B. Textos Básicos de Saúde).

34. Fonseca FCS. Sistemas de informação da atenção à saúde: da fragmentação à interoperabilidade. In: Ministério da Saúde (BR), Secretaria de Atenção à Saúde, Departamento de Atenção Básica. Sistemas de Informação da Atenção à Saúde: contextos históricos, avanços e perspectivas no SUS. Brasília, DF; 2015. p.9-21.

35. Sæbø JI, Kossi EK, Titlestad OH, Tohouri RR, Braa J. Comparing strategies to integrate health information systems following a data warehouse approach in four countries. Inform Technol Dev. 2011;17(1):42-60. https://doi.org/10.1080/02681102.2010.511702

36. Adenuga OA, Kekwaletswe RM, Coleman A. eHealth integration and interoperability issues: towards a solution through enterprise architecture. Health Inf Sci Syst. 2015;3:1. https://doi.org/10.1186/s13755-015-0009-7

37. Kanter AS, Borland R, Barasa M, Iliams-Hauser C, Velez O, Kaonga NN, et al. The importance of using open source technologies and common standards for interoperability within eHealth: perspectives from the Millennium Villages Project. Adv Health Care Manag. 2012;12:189-204. https://doi.org/10.1108/S1474-8231(2012)0000012013

38. Higman S, Dwivedi V, Nsaghurwe A, Busiga M, Rulargirwa HS, Smith D, et al. Designing interoperable health information systems using Enterprise Architecture approach in resourcelimited countries: a literature review. Int J Health Plann Manag. 2019;34(1):e85-99. https://doi.org/10.1002/hpm.2634

39. Liyanage $\mathrm{H}$, Krause $\mathrm{P}$, Lusignan $\mathrm{S}$. Using ontologies to improve semantic interoperability in health data. BMJ Health Care Inform. 2015;22(2):309-15. https://doi.org/10.14236/jhi.v22i2.159

40. Campos CEA. O desafio da integralidade segundo as perspectivas da vigilância da saúde e da saúde da família. Cienc Saude Coletiva. 2003;8(2):569-84. https://doi.org/10.1590/S1413-81232003000200018 